\title{
Identification of process damping for chatter prediction in milling
}

\author{
Charles M. Zheng ${ }^{1}$, Chou-Fu Liang ${ }^{2, a}$, Hai-Yi Cai ${ }^{1}$ and Shui-Shen Zhang ${ }^{1}$ \\ ${ }^{1}$ Quanzhou Institute of Technology, Department of Mechanical Manufacturing and Automation, 362268 Jinjiang, Fujian, China \\ 2,a Fujian Agriculture and Forestry University, College of Transportation and Civil Engineering, 350108 Fuzhou, Fujian, China
}

\begin{abstract}
Traditionally, forecasting stability lobe diagram in milling is limited by complex damping identification procedures, so only structural damping from the impact experiment is used for predicting stability lobe diagram in most milling cases. In this study, by using the mathematical expressions among damping ratio, "critical limiting depth of cut" and "worst spindle speed", it is shown that the predicted "critical limiting depth of cut" based on the structural damping divided by the measured "critical limiting depth of cut" can be approximately equal to the structural damping divided by the total damping. Based on this relationship, it is easy to estimate the total damping or process damping from the experiment within the selected spindle speeds. In practice, this paper presents an easy method for predicting stability lobe diagram using the total damping. At the same time, experiments have confirmed that using the prediction model of total damping can more accurately predict the stability lobe diagram.
\end{abstract}

\section{Introduction}

Damping in the milling process can be divided into two kinds, one is structural damping, and the other is process damping. Structural damping is the energy dissipation mechanism from the cutting tool, fixture, workpiece and machine tool structure, and the process damping is the energy dissipation mechanism from the milling process. Structural damping can be identified through the simple impact testing, but the identification of process damping is difficult and complex in general case.

Process damping has been studied for many years. Early studies have demonstrated that process damping mainly comes from the interference between the flank of cutting tool and the formed waves in milling [1-4], and the spindle speed is lower, the greater the process damping. In the past, the research on the identification of process damping can be generally summarized into two ways. One is to identify process damping from experiments [5-9], and the other is to identify process damping by theoretical modelling [10-14]. But so far, there is rarely a simple and practical identification method to be applied to predict stability lobe diagram in milling, the main reason is that the cutting process of damping is a nonlinear phenomenon [13], and includes some uncertainty [15]. In past studies, most works focus on grasping more damping phenomenon in cutting process, and the identification procedures are more numerous. So, when its application to predict stability lobe diagram, the process will be complicated.

However, the range of selectable spindle speeds is generally not too large in practical milling. Under the limited spindle speeds range condition, presuming the process damping as a constant damping is a reasonable assumption. In the limited spindle speeds range, a simple and practical identification method of process damping for predicting stability lobe diagram is presented as follow section.

\section{Identification of process damping}

A typical stability lobe diagram in milling is shown as Fig. 1. The ordinate corresponding to the lowest position in the figure is called "critical limiting depth of cut", dalim,crit, and its corresponding speed is called "worst spindle speed"; at the same time, the speed corresponding to the peak of the stability lobe diagram is called "best spindle speed".

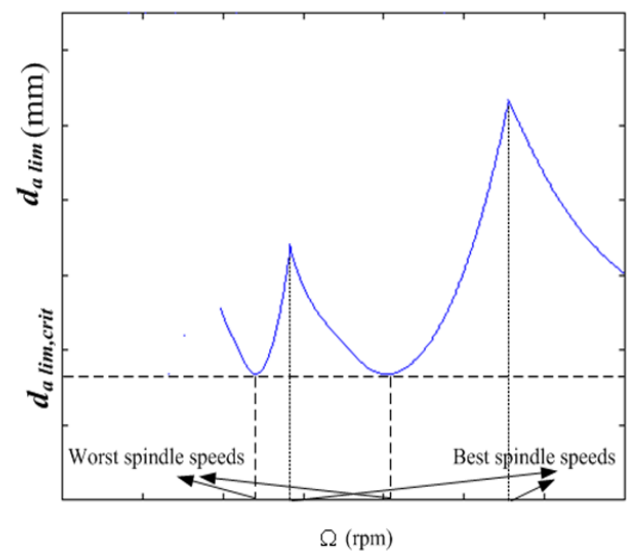

Figure 1. Typical stability lobe diagram in milling

According to the result of eigenvalue analysis by Zheng et al. [16], the mathematical expressions of "critical limiting depth of cut", dalim,crit and the "worst

*Corresponding author: ${ }^{\text {a }}$ Lcf66131@ fafu.edu.cn 
spindle speed" at $n$th lobe of stability lobe diagram, $\Omega_{\text {worst }, n}$ can be written as:

$$
\begin{aligned}
& d a_{\lim , \text { crit }}=\frac{2 \pi}{N k_{t}\left|\lambda_{1}\right|} \frac{k \zeta\left[1+c_{0}{ }^{2}\right]}{c_{0} \cos \phi_{\lambda}-\sin \phi_{\lambda}} \\
& \Omega_{w o r s t, n}=\frac{2 \pi \omega_{n}\left(1+c_{0} \zeta\right) \times 60}{N\left\{\left[\frac{3 \pi}{2}+2 \phi_{\lambda}+\tan ^{-1}\left(\frac{1-c_{0}^{2}}{2 c_{0}}\right)\right]+2 n \pi\right\}}
\end{aligned}
$$

where $\omega_{n}, k$ and $\zeta$ are nature frequency, static stiffness and damping ratio of cutting system, respectively, measured through impact testing. ; $N$ and $k_{t}$ represent flute number of cutter and the tangential specific cutting constant, respectively; $\lambda_{1}, \phi_{\lambda}$ and $c_{0}$ are the radial effect factors which are related to the radial specific cutting constant and radial immersion angle as also stated in [16]. It is noted that damping ratio affects the "critical limiting depth of cut" and the "worst spindle speed" in diffrent ways. Equation (1) shows that the "critical limiting depth of cut" is proportional to damping ratio $\zeta$ while Eq. (2) shows that the "worst spindle speed" is proportional to " $1+c_{0} \zeta$ ". It is shown that damping ratio is less than 0.1 in general milling case, and the value of $c_{0}$ is between 0 and 1[16]. Therefore, it can be concluded that damping ratio has small influence on the "worst spindle speed", which gives a reasonable inference that the predicted "worst spindle speed" base on structural damping is equal to the measured "worst spindle speed", if rest of the cutting parameters remain the same. Based on the important inference, considering the total damping is a combination of structural damping and process damping, the identification of process damping can be desighned as follow steps :

- Identifying the modal parameters of cutting system $\omega_{n}, k$ and $\zeta$ through impact test.

- Identifying tangential specific cutting constant $k_{t}$ and radial specific cutting constant $k_{r}$ using the presented method from [17].

- Calculating the radial effect factors $\lambda_{1}, \phi_{\lambda}$ and $c_{0}$ from [16].

- Substituting the modal parameters, specific cutting constants and radial effect factors into Eq. (1)-(2) to find the "critical limiting depth of cut" dalim,crit,s and the corresponding "worst spindle speed" $\Omega_{\text {worst, }, s}$, based on the structural damping.

- Letting the measured "worst spindle speed" due to total damping, $\Omega_{\text {worst }, n, t}$ is equal to $\Omega_{\text {worst }, n, s}$.

- Finding the "critical limiting depth of cut" due to total damping, dalim,crit,t at the spindle speed $\Omega_{w o r s t, n, t}$ from the cutting test.

- Calculating the total damping $\zeta_{t}$ from the following relation:

$$
d a_{\mathrm{lim}, c r i t, s} / d a_{\mathrm{lim}, c r i t, t}=\zeta / \zeta_{t}
$$

- Calculating the process damping $\zeta_{p}=\zeta_{t}-\zeta$
A flowchart for finding the process damping is presented in Fig. 2.

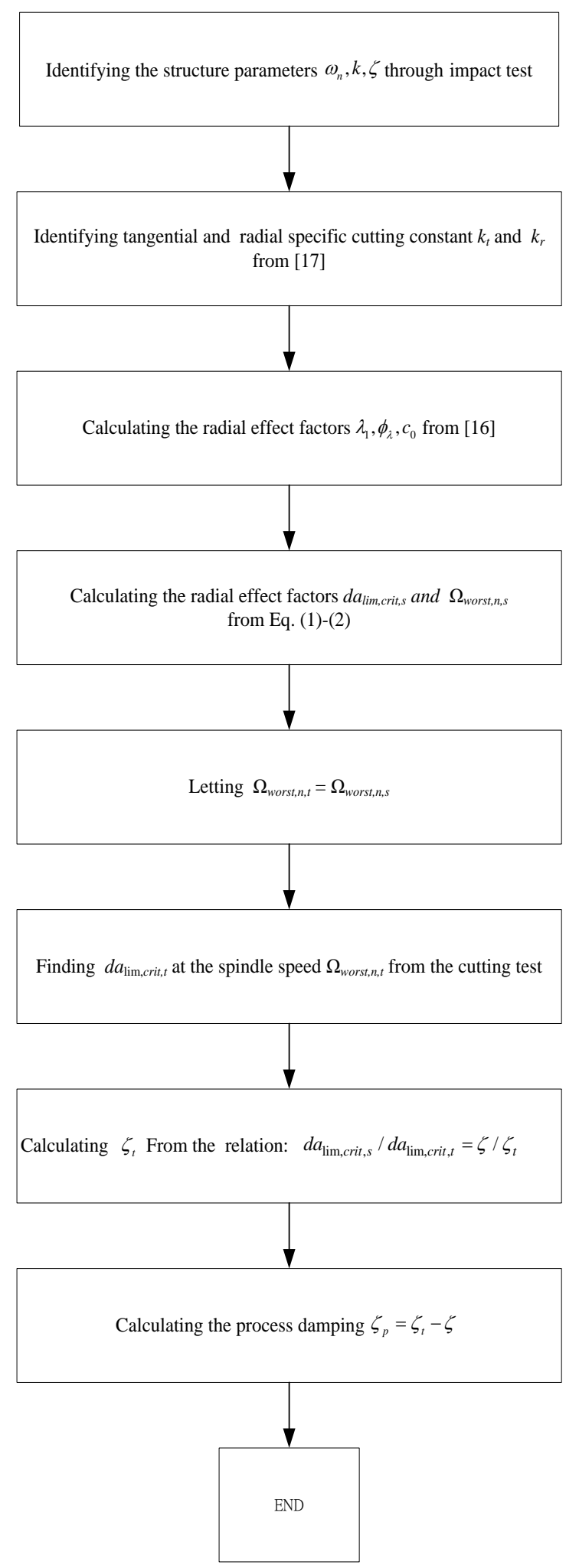

Figure 2. Flowchart for finding the process damping

\section{Experimental Verification}

Milling experiments were carried out with 3-axis vertical milling machine. A two-fluted tungsten carbide end-mill of $20 \mathrm{~mm}$ diameter and 15 degrees helix angle was used in milling the SKD61. Measured signals from the 
accelerometer are used to determine whether chatter has occurred. Note that only signals at the tooth passing frequency and higher harmonics are found in a stable milling, an unstable milling has additional strong signal content at the chatter frequency which is near the nature frequency of the cutting system.

The experiment starts with identifying the modal parameters of cutting system. The dynamics of the milling system were identified for the workpice and cutting tool respectively from impact testing. The real and imaginary parts of the FRFs were calculated to identify the modal parameters using peak-picking method. Identification results shows that the rigidity of workpice is about 100 times higher than the cutting tool, so it is reasonable to treat the workpice as a rigid body and ignore its compliance. With the symmetry of end mill with respect to the spindle axis, the dynamics of the cutting tool is nearly identical in any direction on $\mathrm{X}-\mathrm{Y}$ plane. The most prominent mode of the cutting tool is found at $1200 \mathrm{~Hz}$ and the modal parameters of the 1200 $\mathrm{Hz}$ mode are listed in Table 1, and are used to predict stability lobe diagram.

Table 1. Modal parameters of $1200 \mathrm{~Hz}$ mode identified from impact testing.

\begin{tabular}{|c|c|c|}
\hline $\begin{array}{c}\text { Natural } \\
\text { frequency, } \\
\omega_{n}(\mathrm{~Hz})\end{array}$ & $\begin{array}{c}\text { Stiffness, } \\
\mathrm{k}(\mathrm{N} / \mathrm{m})\end{array}$ & Damping ratio \\
\hline 1200 & $7.4 \times 10^{7}$ & 0.0075 \\
\hline
\end{tabular}

The method for the estimation of specific cutting constant as presented by Wang and Zheng [17] is used in this study. Since the regenerative chatter is only attributed to the shearing mechanism, only the cutting constants related to the shearing mechanism in [17] are used in predicting stability lobe diagram, "critical limiting depth of cut" and "worst spindle speed". The identified cutting constants are listed in Table 2.

Table 2. Cutting constants identified from cutting tests by the method presented in [17].

\begin{tabular}{|c|c|}
\hline \multicolumn{2}{|c|}{ Shearing mechanism } \\
\hline Tangential specific & Radial specific cutting \\
cutting constant & constant \\
$k_{t s}\left(\mathrm{~N} / \mathrm{mm}^{2}\right)$ & $k_{r s}$ \\
\hline 1570 & 0.343 \\
\hline
\end{tabular}

Cutting testing was carried out in down milling with $50 \%$ radial immersion, and thus the radial effect factors $\lambda_{1}, \phi_{\lambda}$ and $c_{0}$ can be found from [16] as table 3 .

Table 3. Radial effect factors for $50 \%$ radial immersion in down milling .

\begin{tabular}{|c|c|c|}
\hline$\lambda_{1}$ & $\phi_{\lambda}$ (Deg.) & $c_{0}$ \\
\hline $0.269-0.58 \mathrm{j}$ & $-47.1^{\circ}$ & 0.48 \\
\hline
\end{tabular}

Substituting the modal parameters, specific cutting constants and radial effect factors into Eq. (1), the corresponding "critical limiting depth of cut" are found to be $1.82 \mathrm{~mm}$. Furthermore, substituting the modal parameters, specific cutting constants and radial effect factors into Eq. (2), the worst spindle speeds can be obtained. According to the experience of operator, the selectable spindle speeds for the 3-axis vertical milling machine in milling SKD61 is between $1900 \mathrm{rpm}$ and $2500 \mathrm{rpm}$. The predicted worst spindle speeds based on structure damping between $1900 \mathrm{rpm}$ and $2500 \mathrm{rpm}$ are calculated and listed in Table 4.

Table 4. The predicted worst spindle speeds based on structure damping between $1900 \mathrm{rpm}$ and $2500 \mathrm{rpm}$

\begin{tabular}{|c|c|c|c|c|c|}
\hline$n$ & 18 & 17 & 16 & 15 & 14 \\
\hline $\begin{array}{c}\Omega_{\text {worst }, n, s} \\
(\mathrm{rpm})\end{array}$ & 1941 & 2051 & 2175 & 2315 & 2474 \\
\hline
\end{tabular}

In order to verify the spindle speeds listed in Table 4 are the worst spindle speeds, ZOS method presented by Budak and Altintas [18] is used to predict the stability lobe diagram using the parameters as listed in Table 1 and Table 2 for $50 \%$ radial immersion in down milling . Through visual inspection in Fig. 3, it can be found that the worst spindle speeds listed in Table 4 agree very well

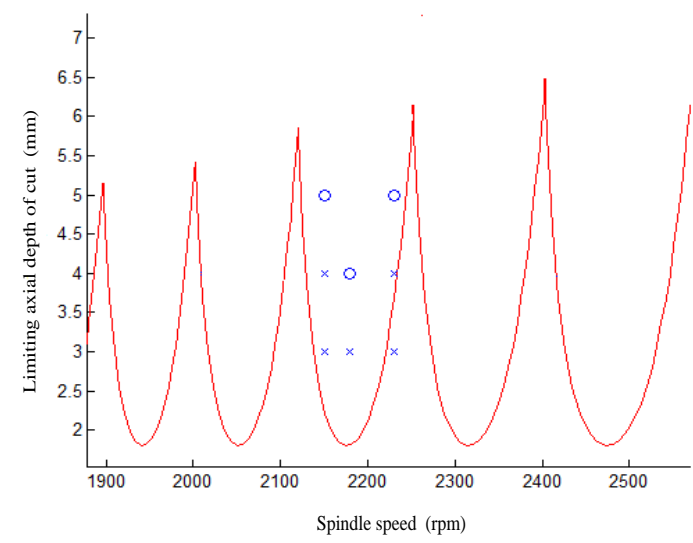

Figure 3. Comparison of experimental results and stability lobe diagram from ZOS [18 using damping ratio $=0.0075$.

with the results from ZOS method. Note that the ZOS method does not provide explicit expressions for the worst spindle speeds and its corresponding critical limiting chip width.

As shown in Fig. 3, although changes of the measured "limiting depth of cut" in the trend agree with predicted results, the measured " critical limiting depth of cut" from milling test at $2175 \mathrm{rpm}$ (the worst spindle speed of the $16^{\text {th }}$ lobe) is about twice the predicted value of $1.82 \mathrm{~mm}$. The inaccurate prediction for critical limiting depth of cut mainly results from the effect of process damping. From Eq. (3), the total damping is found about 0.015 , twice the structure damping, which implies that process damping is about equal to the structure damping. To verify the above reasoning, a new damping ratio of 0.015 is used to predict the stability lobe diagram by ZOS method. The new stability lobe diagram is shown 
in Fig. 4 to agree well with the experimental results. As evidenced by the stability lobe diagrams in Fig. 3 and Fig. 4, as damping ratio increases from 0.0075 to 0.015 , the critical limiting depth of cut doubles while the worst spindle speeds vary slightly as analysed in section 2. In this milling case, comparing worst spindle speed by using total damping, the estimated error of the worst spindle speed by using structure damping is only $0.36 \%$.

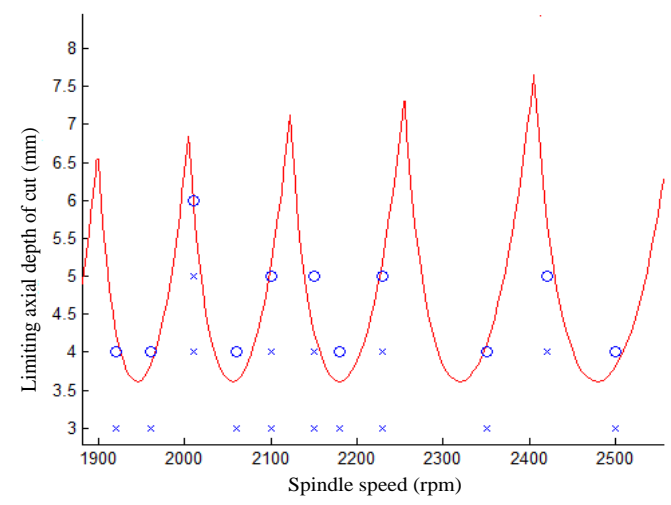

Figure 4. Comparison of experimental results and stability lobe diagram from ZOS [10] using damping ratio $=0.015$.

\section{Conclusions and discussions}

In this study, the two mathematical formulations of "critical limiting depth of cut" and the "worst spindle speed" are used to design the simple and practical procedures for the identification of process damping in milling. There are some conclusions and discussions are summarized as follows :

- Based on the formulation of "critical limiting depth of cut", it can be concluded that the predicted "critical limiting depth of cut" based on the structural damping divided by the measured "critical limiting depth of cut" can be approximately equal to the structural damping divided by the total damping.

- Based on the formulation of "worst spindle speed", it can be concluded that damping ratio has small influence on the "worst spindle speed", which gives a reasonable inference that the "worst spindle speed" predicted by the structural damping is equal to the measured "worst spindle speed" in milling.

In applying the two mathematical formulations to identify the process damping, note that two assumptions are made:

- Comparing the cutting tool, the workpice is assumed as a rigid body. If the compliance of workpice can't be ignored, the presented identification method is not suitable.
- The process damping is assumed as a constant in the limited spindle speeds range. If the range of selectable spindle speeds is large, the process damping can be modified as the function of $\mathrm{n}$ (ordinal number of lobe) for predicting the stability lobe diagram.

\section{References}

1. P.W. Wallace, C. Andrew, Machining forces: Some effects of tool vibration, Journal of Mechanical Engineering Science 7, 152-162 (1965)

2. T.R. Sisson, R.L. Kegg, An explanation of lowspeed chatter effects, Journal of Engineering for Industry 91, 951-958 (1969)

3. J. Peters, P. Vanherck, H.V. Brussel, The measurement of the dynamic cutting coefficient, Annals of the CIRP 21/2, 129-136 (1971)

4. J. Tlusty, Analysis of the state of research in cutting dynamics, Annals of the CIRP 27/2, 583-589 (1978)

5. Y. Altintas, M. Eynian, H. Onozuka, Identification of dynamic cutting force coefficients and chatter stability with process damping, CIRP Ann. Manuf. Technol 57, 371-374 (2008)

6. E. Budak, L.T. Tunc, A new method for identification and modeling of process damping in machining, J. Manuf. Sci. Eng. 131 (2009)

7. L.T. Tunc, E. Budak, Identification and modeling of process damping in milling, J. Manuf. Sci. Eng. 135, 2100-2111 (2013)

8. Y. Kurata, S.D. Merdol, Y. Altintas, , N. Suzuki, E. Shamoto, Chatter stability in turning and milling with in process identified process damping, J. Adv. Mech. Des. Syst. Manuf. 4, 1107-1118 (2010)

9. A.R. Yusoff, , S. Turner, C.M. Taylor, N.D. Sims, The role of tool geometry in process damped milling, Int. J. Adv. Manuf. Technol. 50, 883-895 (2010)

10. D.W. Wu, A new approach of formulating the transfer function for dynamic cutting processes, , J. Manuf. Sci. Eng. 111, 37-47 (1989)

11. R.Y. Chiou, S.Y. Liang, Chatter stability of a slender cutting tool in turning with tool wear effect, Int. J. Mach. Tools Manuf. 38, 315-327 (1998)

12. C.Y. Huang, J.J. Wang, Mechanistic modeling of process damping in peripheral milling, J. Manuf. Sci. Eng. 129, 12-20 (2007)

13. K. Ahmadi, F. Ismail, Analytical stability lobes including nonlinear process damping effect on machining chatter, Int. J. Mach. Tools Manuf. 51, 296-308 (2011)

14. C. Cao, X.M. Zhang, H. Ding, An improved algorithm for cutting stability estimation considering process damping. Int. J. Adv. Manuf. Technol. 88, 2029-2038 (2017)

15. J.M. Karandikar, C.T. Tyler, T.L. Schmitz, Process Damping Coefficient Identification using Bayesian Inference, Proceedings of NAMRC/SME 41, 1-11 (2013) 
16. C.M. Zheng, J.J. Wang and, C.F. Sung, Analytical prediction of the critical depth of cut and worst spindle speeds for chatter in end milling, ASME Journal of Manufacturing Science and Engineering, 136 (2013)

17. J.J. Wang, C.M. Zheng, An analytical force model with shearing and ploughing mechanisms for end milling, International Journal of Machine Tools \& Manufacture 42, 761-771 (2002)

18. E. Budak, Y. Altintas, An Analytical Prediction of Chatter Stability in Milling - Part I: General Formulation, ASME Journal of Dynamic Systems, Measurement and Control 120, 22-30 (1998) 